\title{
ON THE KOSZUL COHOMOLOGY OF CANONICAL AND PRYM-CANONICAL BINARY CURVES
}

\author{
ELISABETTA COLOMBO AND PAOLA FREDIANI
}

\begin{abstract}
In this paper we study Koszul cohomology and the Green and Prym-Green conjectures for canonical and Prym-canonical binary curves. We prove that if property $N_{p}$ holds for a canonical or a Prym-canonical binary curve of genus $g$ then it holds for a generic canonical or Prym-canonical binary curve of genus $g+1$. We also verify the Green and Prym-Green conjectures for generic canonical and Prym-canonical binary curves of low genus $(6 \leq g \leq 15, g \neq 8$ for Prym-canonical and $3 \leq g \leq 12$ for canonical).
\end{abstract}

\section{INTRODUCTION}

Let $C$ be a smooth curve, $L$ a line bundle and $\mathcal{F}$ a coherent sheaf on $C$. We recall that the Koszul cohomology group $K_{p, q}(C, \mathcal{F}, L)$ is the middle term cohomology of the complex:

$$
\Lambda^{p+1} H^{0}(L) \otimes H^{0}\left(\mathcal{F} \otimes L^{q-1}\right) \stackrel{d_{p+1} q-1}{\rightarrow} \Lambda^{p} H^{0}(L) \otimes H^{0}\left(\mathcal{F} \otimes L^{q}\right) \stackrel{d_{p q} q}{\rightarrow} \Lambda^{p-1} H^{0}(L) \otimes H^{0}\left(\mathcal{F} \otimes L^{q+1}\right)
$$

where

$$
d_{p, q}\left(s_{1} \wedge \ldots \wedge s_{p} \otimes u\right):=\sum_{l=1}^{p}(-1)^{l} s_{1} \wedge \ldots \wedge \hat{s}_{l} \wedge \ldots \wedge s_{p} \otimes\left(s_{l} u\right) .
$$

If $\mathcal{F}=\mathcal{O}_{C}$ the groups $K_{p, q}\left(C, \mathcal{O}_{C}, L\right)$ are denoted by $K_{p, q}(C, L)$. The Koszul cohomology theory has been introduced in [11] and has been extensively studied in particular in the case of the canonical bundle. We recall that Green and Lazarsfeld ([11]) proved that for any smooth curve $C$ of genus $g$ and Clifford index $c, K_{g-c-2,1}\left(C, K_{C}\right) \neq 0$. Green's conjecture says that this result is sharp i.e. $K_{p, 1}\left(C, K_{C}\right)=0$ for all $p \geq g-c-1$. The Clifford index for a general curve is $\left[\frac{g-1}{2}\right]$, so generic Green's conjecture says that $K_{p, 1}\left(C, K_{C}\right)=0$ for all $p \geq\left[\frac{g}{2}\right]$, or equivalently, by duality, $K_{p, 2}\left(C, K_{C}\right)=0$, i.e. property $N_{p}$ holds, for all $p \leq\left[\frac{g-3}{2}\right]$. Generic Green's conjecture has been proved by Voisin in [20, 21]. Green's conjecture has also been verified for curves of odd genus and maximal Clifford index ([21], 12]), for general curves of given gonality ([20, [18 [17]), for curves on $K 3$-surfaces ([20, [21], [1]), and in other cases (see [2]).

Another interesting case is when the line bundle is Prym canonical, $L=K_{C} \otimes A$ where $A$ is a non trivial 2 torsion line bundle. This case has been studied in [8], where the Prym-Green conjecture has been stated. This is an analogue of the Green conjecture for general curves, namely it says that for a general Prym-canonical curve $\left(C, K_{C} \otimes A\right)$, we have $K_{p, 2}\left(C, K_{C} \otimes A\right)=0$, i.e. property $N_{p}$ holds, for all $p \leq\left[\frac{g}{2}-3\right]$. Prop.3.1 of $[8]$ shows that for any $\left(C, K_{C} \otimes A\right)$ and $p>\left[\frac{g}{2}-3\right], K_{p, 2}\left(C, K_{C} \otimes A\right) \neq 0$.

Debarre in 7 proved that a generic Prym-canonical curve of genus $g \geq 6$ is projectively normal (property $N_{0}$ ) and for $g \geq 9$ its ideal is generated by quadrics (property $N_{1}$ ).

In [5] the Prym-Green conjecture is proved for genus $g=10,12,14$ by degeneration to irreducible nodal curves and computation with Macaulay2. In a private communication Gavril Farkas told us that they could verify the conjecture also for $g=18,20$. The computations made in 5 for genus 8 and 16 suggest that the Prym-Green conjecture may be false for genus which is a multiple of 8 or perhaps a power of 2. The possible failure of the Prym-Green conjecture in genus 8 is extensively discussed in the last section of [5], where a geometric interpretation of this phenomenon is given.

In this paper we study Koszul cohomology and the Green and Prym-Green conjectures for canonical and Prym-canonical binary curves. Recall that a binary curve of genus $g$ is a stable curve consisting of two rational components $C_{j}, j=1,2$ meeting transversally at $g+1$ points. The canonical and Prym-canonical models of binary curves that we analyze are the one used in [3] and [6] and described

\footnotetext{
${ }^{1}$ Partially supported by PRIN 2009: "Moduli, strutture geometriche e loro applicazioni" and by INdAM (GNSAGA) AMS Subject classification: 14H10, 14H40, 13D02.
} 
in the next section. The main result of the paper (Theorem (3.2)) says that if property $N_{p}$ holds for a Prym-canonical binary curve of genus $g$ then it holds for a generic Prym-canonical binary curve of genus $g+1$. In particular, if the Prym-Green conjecture is true for a Prym-canonical binary curve of genus $g=2 k$, then it is true for a general Prym-canonical binary curve of genus $g=2 k+1$.

Moreover we verify the conjecture by a direct computation for $g=6,9,10,12,14$ (see Corollary (3.4)).

As a consequence, we show that the generic Prym-canonical curve of genus $g$ satisfies property $N_{0}$ for $g \geq 6$, property $N_{1}$ for $g \geq 9$ (already shown by Debarre), property $N_{2}$ for $g \geq 10$, property $N_{3}$ for $g \geq 12$ and property $N_{4}$ for $g \geq 14$ (Corollary (3.4)).

For $g=8$ and $g=16$ our computations on Prym-canonical binary curves also suggest that PrymGreen conjecture's might fail, in fact in our examples we find that $K_{\frac{g}{2}-3,2}\left(C, K_{C} \otimes A\right)=1$ both for $g=8$ and $g=16$ (see Remark (3.5)).

An analogous result of Theorem (3.2) is proven for canonically embedded binary curves (Theorem (4.2) ), where we show that if property $N_{p}$ holds for a canonical binary curve of genus $g$, then the same property holds for a general canonical binary curve of genus $g+1$. In particular, if the Green conjecture is true for a canonical binary curve of genus $g=2 k-1$, then it is true for a general canonical binary curve of genus $g=2 k$.

Theorem (3.2) and analogous computations with maple in genus $g=3,5,7,9,11$, imply that for a general canonical binary curve, if $g \geq 3$, then propery $N_{0}$ holds (see also 3 section 2 ), if $g \geq 5$, then propery $N_{1}$ holds, if $g \geq 7$, then propery $N_{2}$ holds, if $g \geq 9$, then property $N_{3}$ holds, and if $g \geq 11$, then property $N_{4}$ holds.

Acknowledgments. We thank Riccardo Murri for having been so kind to do for us the computer computations in $g=14,16$.

\section{CAnonical and Prym-CANONicAl Binary CURVES}

2.1. Construction of canonical binary curves. Recall that a binary curve of genus $g$ is a stable curve consisting of two rational components $C_{j}, j=1,2$ meeting transversally at $g+1$ points. Moreover, $H^{0}\left(C, \omega_{C}\right)$ has dimension $g$ and the restriction of $\omega_{C}$ to the component $C_{j}$ is $K_{C_{j}}\left(D_{j}\right)$ where $D_{j}$ is the divisor of nodes on $C_{j}$. Since $K_{C_{j}}\left(D_{j}\right) \cong \mathcal{O}_{\mathbb{P}^{1}}(g-1)$ we observe that the components are embedded by the complete linear system $\left|\mathcal{O}_{\mathbb{P}^{1}}(g-1)\right|$ in $\mathbb{P}^{g-1}$.

Following [3], we assume that the first $g$ nodes are $P_{i}=(0, \ldots, 0,1,0, \ldots 0)$, with 1 at the $i$-th place, $i=1, \ldots, g$. Then we can assume that $C_{j}$ is the image of the map

$$
\begin{gathered}
\phi_{j}: \mathbb{P}^{1} \rightarrow \mathbb{P}^{g-1}, j=1,2 \\
\phi_{j}(t, u):=\left[\frac{M_{j}(t, u)}{\left(t-a_{1, j} u\right)}, \ldots, \frac{M_{j}(t, u)}{\left(t-a_{g-1, j} u\right)}\right]
\end{gathered}
$$

with $M_{j}(t, u):=\prod_{r=1}^{g}\left(t-a_{r, j} u\right), j=1,2$ and $\phi_{j}\left(\left[a_{l, j}, 1\right]\right)=P_{l}, l=1, \ldots, g$.

We see that the remaining node is the point $P_{g+1}:=[1, \ldots, 1]$ and it is the image of $[1,0]$ through the maps $\phi_{j}, j=1,2$. One can easily check that, for generic values of the $a_{i, j}$ 's, $C=C_{1} \cup C_{2}$ is a canonically embedded binary curve.

2.2. Construction of Prym-canonical binary curves. Let $C$ be a binary curve of genus $g$, and $A \in$ $P i c^{0}(C)$ a nontrivial line bundle. Then $H^{0}\left(C, \omega_{C} \otimes A\right)$ has dimension $g-1$ and the restriction of $\omega_{C} \otimes A$ to the component $C_{j}$ is $K_{C_{j}}\left(D_{j}\right)$ where $D_{j}$ is the divisor of nodes on $C_{j}$. Since $K_{C_{j}}\left(D_{j}\right) \cong \mathcal{O}_{\mathbb{P}^{1}}(g-1)$, the components are embedded by a linear subsystem of $\mathcal{O}_{\mathbb{P}^{1}}(g-1)$, hence they are projections from a point of rational normal curves in $\mathbb{P}^{g-1}$. Viceversa, let us take 2 rational curves embedded in $\mathbb{P}^{g-2}$ by non complete linear systems of degree $g-1$ intersecting transversally at $g+1$ points. Then their union $C$ is a binary curve of genus $g$ embedded either by a linear subsystem of $\omega_{C}$ or by a complete linear system $\left|\omega_{C} \otimes A\right|$, where $A \in P i c^{0}(C)$ is nontrivial (see e.g. [4, Lemma 10). In 6] (Lemma 3.1) we constructed a binary curve $C$ embedded in $\mathbb{P}^{g-2}$ by a linear system $\left|\omega_{C} \otimes A\right|$ with $A^{\otimes 2} \cong \mathcal{O}_{C}$, and 
$A$ is non trivial. Let us now recall this construction and denote a binary curve with this embedding a Prym-canonical binary curve.

Assume that the first $g-1$ nodes, are $P_{i}=(0, \ldots, 0,1,0, \ldots 0)$ with 1 at the $i$-th place, $i=1, \ldots, g-1$, the remaining two nodes are $P_{g}:=\left[t_{1}, \ldots, t_{g-1}\right]$ with $t_{i}=0$ for $i=1, \ldots,\left[\frac{g}{2}\right], t_{i}=1$, for $i=\left[\frac{g}{2}\right]+1, \ldots, g-1$. and $P_{g+1}:=\left[s_{1}, \ldots, s_{g-1}\right]$ with $s_{i}=1$ for $i=1, \ldots,\left[\frac{g}{2}\right], s_{i}=0$, for $i=\left[\frac{g}{2}\right]+1, \ldots, g-1$.

Then the component $C_{j}$ is the image of the map

$$
\begin{gathered}
\phi_{j}: \mathbb{P}^{1} \rightarrow \mathbb{P}^{g-2}, j=1,2, \text { where } \\
\phi_{1}(t, u):=\left[\frac{t M_{1}(t, u)}{\left(t-a_{1,1} u\right)}, \ldots, \frac{t M_{1}(t, u)}{\left(t-a_{k, 1} u\right)}, \frac{-M_{1}(t, u) d_{1} a_{k+1,1} u}{A_{1}\left(t-a_{k+1,1} u\right)}, \ldots, \frac{-M_{1}(t, u) d_{1} a_{g-1,1} u}{A_{1}\left(t-a_{g-1,1} u\right)}\right] \\
\phi_{2}(t, u):=\left[\frac{t M_{2}(t, u)}{\left(t-a_{1,2} u\right)}, \ldots, \frac{t M_{2}(t, u)}{\left(t-a_{k, 2} u\right)}, \frac{-M_{2}(t, u) d_{2} a_{k+1,2} u}{A_{2}\left(t-a_{k+1,2} u\right)}, \ldots, \frac{-M_{2}(t, u) d_{2} a_{g-1,2} u}{A_{2}\left(t-a_{g-1,2} u\right)}\right]
\end{gathered}
$$

with $k:=\left[\frac{g}{2}\right], M_{j}(t, u):=\prod_{r=1}^{g-1}\left(t-a_{r, j} u\right)$, and $A_{j}=\prod_{i=1}^{g-1} a_{i, j}, j=1,2, d_{2}$ is a nonzero constant and $d_{1}=\frac{-d_{2} A_{1}}{A_{2}}$. Notice that we have $\phi_{j}\left(\left[a_{l, j}, 1\right]\right)=P_{l}, l=1, \ldots, g-1, \phi_{j}([0,1])=P_{g}, \phi_{j}([1,0])=P_{g+1}$, $j=1,2$. In Lemma 3.1 of [6] we proved that for a general choice of $a_{i, j}$ 's, $C=C_{1} \cup C_{2}$ is a binary curve embedded in $\mathbb{P}^{g-2}$ by a linear system $\left|\omega_{C} \otimes A\right|$ with $A^{\otimes 2} \cong \mathcal{O}_{C}$ and $A$ nontrivial. In fact, recall that $\operatorname{Pic}^{0}(C) \cong \mathbb{C}^{* g} \cong \mathbb{C}^{* g+1} / \mathbb{C}^{*}$, where $\mathbb{C}^{*}$ acts diagonally, and in Lemma 3.1 of $[\underline{6}$ it is shown and our line bundle $A$ corresponds to the element $\left[\left(h_{1}, \ldots, h_{g+1}\right)\right] \in \mathbb{C}^{* g+1} / \mathbb{C}^{*}$, where $h_{i}=1$, for $i<\left[\frac{g}{2}\right]+1$, $h_{i}=-1$, for $i=\left[\frac{g}{2}\right]+1, \ldots, g-1, h_{g}=-1, h_{g+1}=1$, so in particular $A$ is of 2 -torsion.

\section{Property $N_{p}$ For Prym-CANonical Binary CURves}

Let $C \subset \mathbb{P}^{g-2}$ be a Prym-canonical binary curve embedded by $\omega_{C} \otimes A$, with $A^{\otimes 2} \cong \mathcal{O}_{C}$, as in (3). In this section we study the Koszul cohomology for these curves, in particular we investigate property $N_{p}$, i.e. the vanishing of $K_{p, 2}\left(C, K_{C} \otimes A\right)$. Since by duality ([11, see also 9 prop.1.4) we have $K_{p, 2}\left(C, K_{C} \otimes A\right) \cong K_{g-3-p, 0}\left(C, K_{C}, K_{C} \otimes A\right)^{\vee}$, this vanishing is equivalent to the injectivity of the Koszul map

$$
F_{g-3-p}: \Lambda^{g-3-p} H^{0}\left(C, \omega_{C} \otimes A\right) \otimes H^{0}\left(C, \omega_{C}\right) \rightarrow \Lambda^{g-4-p} H^{0}\left(C, \omega_{C} \otimes A\right) \otimes H^{0}\left(C, \omega_{C}^{2} \otimes A\right) .
$$

Our strategy is to compare this map with analogous Koszul maps for a partial normalization of the curve $C$ at one node and possibly use induction on the genus.

To this end, let us introduce some notation: set $k:=\left[\frac{g}{2}\right]$ and denote by $\tilde{C}_{r}$ the partial normalization of $C$ at the node $P_{r}$ with $r \leq k$ if $g=2 k, r \geq k+1$ if $g=2 k+1$. This choice of the node is necessary in order to obtain the Prym-canonical model for the curve $\tilde{C}_{r}$. In fact, observe that in this way, for a general choice of the $a_{i, j}$ 's, the projection from $P_{r}$ sends the curve $C$ to the Prym-canonical model of $\tilde{C}_{r}$ in $\mathbb{P}^{g-3}$ given by the line bundle $K_{\tilde{C}_{r}} \otimes A_{r}^{\prime}$ where $A_{r}^{\prime}$ corresponds to the point $\left(h_{1}^{\prime}, \ldots, h_{g-1}^{\prime}, 1\right) \in \mathbb{C}^{* g} / \mathbb{C}^{*}$, with $h_{i}^{\prime}=1$ for $i \leq\left[\frac{g-1}{2}\right], h_{i}^{\prime}=-1$ for $i=\left[\frac{g-1}{2}\right]+1, \ldots, g-1$, as described above. In fact $\left(\tilde{C}_{r}, A_{r}^{\prime}\right)$ is parametrized by $a_{i, j}^{\prime}=a_{i, j}$ for $i \leq r-1, j=1,2, a_{i, j}^{\prime}=a_{i+1, j}$ for $i \geq r, j=1,2$. So if we set $d_{j}^{\prime}:=\frac{d_{j}}{a_{r, j}}$, $j=1,2$, we clearly have a pair $\left(\tilde{C}_{r}, A_{r}^{\prime}\right)$ as in (3). For simplicity let us choose $d_{2}=1$, so $d_{1}=-\frac{A_{1}}{A_{2}}$, hence $d_{2}^{\prime}:=\frac{1}{a_{r, 2}}, d_{1}^{\prime}:=-\frac{A_{1}}{A_{2} a_{r, 1}}$.

To simplify the notation, set $T_{g}:=H^{0}\left(C, \omega_{C} \otimes A\right), H_{g}:=H^{0}\left(C, \omega_{C}\right), B_{g}:=H^{0}\left(C, \omega_{C}^{2} \otimes A\right)$. Denote by $\left\{t_{1}, \ldots, t_{g-1}\right\}$ the basis of $T_{g}$ given by the coordinate hyperplane sections in $\mathbb{P}^{g-2} \cong \mathbb{P}\left(T_{g}^{\vee}\right)$ and by $\left\{s_{1}, \ldots, s_{g}\right\}$ the basis of $H_{g}$ given by the coordinate hyperplane sections in $\mathbb{P}^{g-1} \cong \mathbb{P}\left(H_{g}^{\vee}\right)$. $T_{g-1, r}:=$ $H^{0}\left(\tilde{C}_{r}, \omega_{\tilde{C}_{r}} \otimes A_{r}^{\prime}\right), H_{g-1, r}:=H^{0}\left(\tilde{C}_{r}, \omega_{\tilde{C}_{r}}\right), B_{g-1, r}:=H^{0}\left(\tilde{C}_{r}, \omega_{\tilde{C}_{r}}^{2} \otimes A_{r}^{\prime}\right)$. Denote by $\left\{t_{1}^{\prime}, \ldots, t_{g-2}^{\prime}\right\}$ the basis of $T_{g-1, r}$ given by the coordinate hyperplane sections in $\mathbb{P}^{g-3} \cong \mathbb{P}\left(T_{g-1, r}^{\vee}\right)$ and by $\left\{s_{1}^{\prime}, \ldots, s_{g-1}^{\prime}\right\}$ the basis of $H_{g-1, r}$ given by the coordinate hyperplane sections in $\mathbb{P}^{g-2} \cong \mathbb{P}\left(H_{g-1, r}^{\vee}\right)$.

We have the following injections:

$$
T_{g-1, r} \stackrel{I_{r}}{\longrightarrow} T_{g}, t_{i}^{\prime} \mapsto t_{i} \text { for } i \leq r-1, t_{i}^{\prime} \mapsto t_{i+1} \text { for } i \geq r,
$$




$$
H_{g-1, r} \stackrel{J_{r}}{\longrightarrow} H_{g}, s_{i}^{\prime} \mapsto s_{i} \text { for } i \leq r-1, s_{i}^{\prime} \mapsto s_{i+1} \text { for } i \geq r
$$

Clearly these maps induce an injective map

$$
B_{g-1, r} \stackrel{L_{r}}{\longrightarrow} B_{g},
$$

which on the set of generators of $B_{g-1, r}$ given by $t_{i}^{\prime} s_{j}^{\prime}, i=1, \ldots, g-2, j=1, \ldots, g-1$ is given by $t_{i}^{\prime} s_{j}^{\prime} \mapsto I_{r}\left(t_{i}^{\prime}\right) J_{r}\left(s_{j}^{\prime}\right)$. We claim that this map is well defined and injective by the definition of the $t_{i}^{\prime}$ 's and $s_{j}^{\prime}$ 's. In fact the restriction of $\sum \alpha_{i, j} t_{i}^{\prime} s_{j}^{\prime}$ to the two rational components of $\tilde{C}_{r}$ yields two polynomials $Q_{1}$ and $Q_{2}$. On the other hand we have $\left(\sum \alpha_{i, j} I_{r}\left(t_{i}^{\prime}\right) J_{r}\left(s_{j}^{\prime}\right)\right)_{\mid C_{i}}=\left(t-a_{r, i}\right)^{2} Q_{i}$, hence $L_{r}$ is well defined and injective. We finally have a map

$$
\Lambda^{l-1} T_{g-1, r} \stackrel{\wedge t_{r}}{\longrightarrow} \Lambda^{l} T_{g},
$$

where by $\wedge t_{r}$ we indicate the composition of the natural map induced by $I_{r}$ at the level of the $(l-1)$-th exterior power $\Lambda^{l-1} T_{g-1, r} \rightarrow \Lambda^{l-1} T_{g}$ composed by the wedge product with $t_{r}, \Lambda^{l-1} T_{g} \stackrel{\wedge t_{r}}{\longrightarrow} \Lambda^{l} T_{g}$.

As in (41), denote by $F_{l}: \Lambda^{l} T_{g} \otimes H_{g} \rightarrow \Lambda^{l-1} T_{g} \otimes B_{g}$ the Koszul map.

We have the following commutative diagram

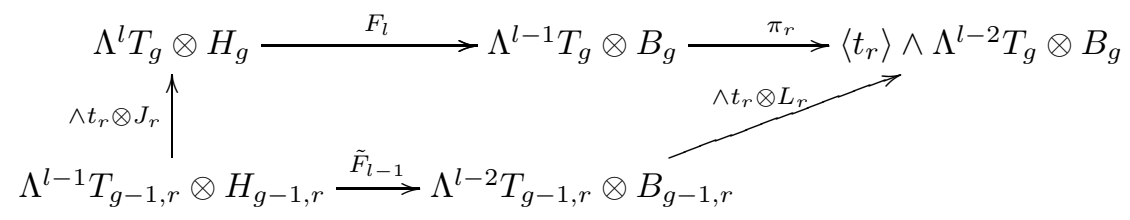

From now on, given a multi-index $I=\left(i_{1}, \ldots, i_{l}\right)$ we denote by $t_{I}:=t_{i_{1}} \wedge \ldots \wedge t_{i_{l}}$.

To study the injectivity of the maps $F_{l}$, a preliminary reduction comes from the following

Lemma 3.1. Let $W \subset \Lambda^{l} T_{g} \otimes H_{g}$ be the subspace generated by the elements of the form $t_{I} \otimes s_{j}$, where $j \notin I$. Then the kernel of the Koszul map $F_{l}: \Lambda^{l} T_{g} \otimes H_{g} \rightarrow \Lambda^{l-1} T_{g} \otimes B_{g}$ is contained in $W$.

Proof. Assume that $v \in \Lambda^{l} T_{g} \otimes H_{g}, v=\sum_{I,|I|=l} \sum_{j=1 \ldots g} \lambda_{j}^{I} t_{I} \otimes s_{j}$ is such that $F_{l}(v)=0 . \quad F_{l}(v)=$ $\sum_{J,|J|=l-1} \sum_{I=J \cup\{m\}} \sum_{j=1 \ldots g} \lambda_{j}^{I} \epsilon(I, J) t_{J} \otimes t_{m} s_{j}=0$, where $\epsilon(I, J)= \pm 1$, depending on the position of $m$ in the multi-index $I=J \cup\{m\}$. Then if we fix a multi-index $J$ with $|J|=l-1$, we must have $\sum_{m} \sum_{j=1 \ldots g} \lambda_{j}^{J \cup\{m\}} \epsilon(J \cup\{m\}, J) t_{J} \otimes t_{m} s_{j}=0$ and therefore

$$
\sigma_{J}:=\sum_{m} \sum_{j=1 \ldots g} \lambda_{j}^{J \cup\{m\}} \epsilon(J \cup\{m\}, J) t_{m} s_{j}=0 .
$$

So we have $\sigma_{J \mid C_{1}} \equiv 0$, namely, if we denote by $P_{1}(t):=t \cdot M_{1}(t, 1)$, as in (3), we have

$$
\begin{gathered}
\sum_{j=1 \ldots g} \sum_{m \leq k} \lambda_{j}^{J \cup\{m\}} \epsilon(J \cup\{m\}, J) \frac{P_{1}(t)}{t-a_{m, 1}} \frac{P_{1}(t)}{t-a_{j, 1}} \\
+\sum_{j=1 \ldots g} \sum_{m \geq k+1} \lambda_{j}^{J \cup\{m\}} \epsilon(J \cup\{m\}, J) \frac{P_{1}(t) a_{m, 1}}{A_{2} t\left(t-a_{m, 1}\right)} \frac{P_{1}(t)}{t-a_{j, 1}}=0
\end{gathered}
$$

If we evaluate in $t=a_{m, 1}$, there remains only one term in the sum, namely the one with $j=m$, and hence we have

$$
\begin{gathered}
\lambda_{m}^{J \cup\{m\}} \epsilon(J \cup\{m\}, J) a_{m, 1}^{2} \cdot \prod_{r \neq m, r=1 \ldots g-1}\left(a_{m, 1}-a_{r, 1}\right)^{2}=0, \text { if } m \leq k, \\
\lambda_{m}^{J \cup\{m\}} \epsilon(J \cup\{m\}, J) \frac{a_{m, 1}^{2}}{A_{2}} \cdot \prod_{r \neq m, r=1 \ldots g-1}\left(a_{m, 1}-a_{r, 1}\right)^{2}=0, \text { if } m \geq k+1,
\end{gathered}
$$

hence we have $\lambda_{m}^{J \cup\{m\}}=0$ for all $m$. 
Since this holds for every multi-index $J$ of cardinality $l-1$, we have shown that we can write $v=\sum_{I,|I|=l} \sum_{j=1 \ldots g, j \notin I} \lambda_{j}^{I} t_{I} \otimes s_{j}$.

We can now state and prove our main result.

Theorem 3.2. Assume that $g=2 k$, or $g=2 k+1$ and take an integer $p \leq k-3$. If property $N_{p}$ holds for a binary curve $\tilde{C}$ of genus $g-1$ embedded in $\mathbb{P}^{g-3}$ by $\left|\omega_{\tilde{C}} \otimes A^{\prime}\right|$ as in (3) for a generic choice of the parameters $a_{i, j}^{\prime}$, then it holds for all binary curves $C$ of genus $g$ embedded in $\mathbb{P}^{g-2}$ by $\left|\omega_{C} \otimes A\right|$ as in (3) for a generic choice of the $a_{i, j}$ 's.

Proof. We want to prove that $K_{p, 2}\left(C, K_{C} \otimes A\right)=0$ for a binary curve of genus $g$ and we know that $K_{p, 2}\left(\tilde{C}_{r}, K_{\tilde{C}_{r}} \otimes A_{r}^{\prime}\right)=0$, for the curve $\tilde{C}_{r}$ which is obtained from $C$ by projection from $P_{r}$ with $r \geq k+1$ if $g=2 k+1, r \leq k$ if $g=2 k$.

By duality, $K_{p, 2}\left(C, K_{C} \otimes A\right) \cong K_{g-3-p, 0}\left(C, K_{C}, K_{C} \otimes A\right)^{\vee}$, so the statement is equivalent to prove injectivity of the Koszul map

$$
F_{g-3-p}: \Lambda^{g-3-p} T_{g} \otimes H_{g} \rightarrow \Lambda^{g-4-p} T_{g} \otimes B_{g} .
$$

By assumption we know injectivity of the map

$$
\tilde{F}_{g-4-p}: \Lambda^{g-4-p} T_{g-1, r} \otimes H_{g-1, r} \rightarrow \Lambda^{g-5-p} T_{g-1, r} \otimes B_{g-1, r} .
$$

For simplicity let us denote by $l:=g-3-p$.

Assume first of all that $g=2 k+1$ and consider the projection of $C$ from $P_{g-1}$.

Recall that by Lemma (3.1) we can reduce to prove injectivity of $F_{l}$ restricted the subspace $W$ generated by such $T_{I} \otimes s_{j}$ with $j \notin I$. Note that we can decompose $W$ as $W:=X_{g-1} \oplus Y_{g-1}$, where $X_{g-1}$ is the intersection with $W$ of the image of the map $\wedge t_{g-1} \otimes J_{g-1}$ in diagram (9) and $Y_{g-1}$ is the subspace of $W$ generated by such $t_{I} \otimes s_{j}$ with $g-1 \notin I$ and $j \notin I$ :

$$
X_{g-1}=\left\langle t_{g-1} \wedge t_{J} \otimes s_{j} \mid j \notin J\right\rangle, Y_{g-1}=\left\langle t_{I} \otimes s_{j} \mid j, g-1 \notin I\right\rangle
$$

Assume now that $F_{l}\left(x_{g-1}+y_{g-1}\right)=0$, where $x_{g-1} \in X_{g-1}, y_{g-1} \in Y_{g-1}$. Then we have $0=$ $\pi_{g-1} \circ F_{l}\left(x_{g-1}+y_{g-1}\right)=\pi_{g-1} \circ F_{l}\left(x_{g-1}\right)=\left(\wedge t_{g-1} \otimes L_{g-1}\right) \circ \tilde{F}_{l-1}\left(x_{g-1}\right)$, by the commutativity of diagram (9). Hence $x_{g-1}=0$, since by induction we are assuming that $\tilde{F}_{l-1}$ is injective. So we have reduced to prove injectivity of $F_{l}$ restricted to $Y_{g-1}$.

Now consider the projection of $C$ from the point $P_{g-2}$.

Set

$$
Y_{g-2}^{\prime}=\left\langle t_{J}^{\prime} \otimes s_{j}^{\prime} \mid j, g-2 \notin J\right\rangle \subset \Lambda^{l-1} T_{g-1, g-2} \otimes H_{g-1, g-2}
$$

Observe that the image $X_{g-2}:=\left(\wedge t_{g-2} \otimes J_{g-2}\right)\left(Y_{g-2}^{\prime}\right)$ is contained in $Y_{g-1}$ and in fact

$$
X_{g-2}=\left\langle t_{g-2} \wedge t_{J} \otimes s_{j} \mid j, g-1 \notin J\right\rangle .
$$

So we have $Y_{g-1}=X_{g-2} \oplus Y_{g-2}$, where $Y_{g-2}$ is the subspace of $Y_{g-1}$ generated by those elements of the form $t_{I} \otimes s_{j}$ where $g-2, g-1, j \notin I$. We have the following commutative diagram

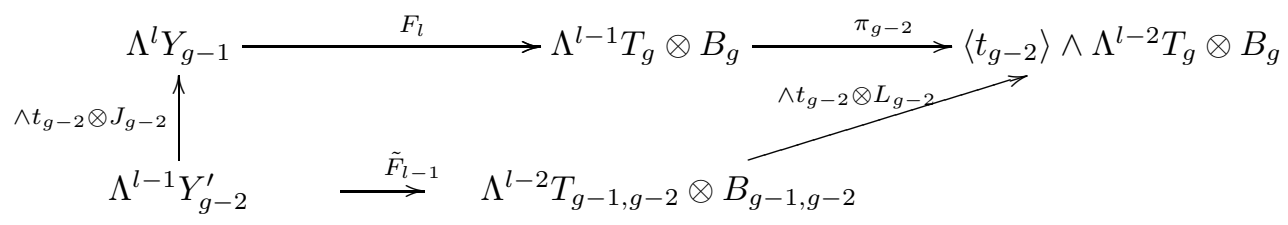

Assume that $v=x_{g-2}+y_{g-2} \in Y_{g-1}=X_{g-2} \oplus Y_{g-2}$ is such that $F_{l}(v)=0$, then we have $0=$ $\pi_{g-2} \circ F_{l}\left(x_{g-2}+y_{g-2}\right)=\pi_{g-2} \circ F_{l}\left(x_{g-2}\right)$. So $0=\tilde{F}_{l-1}\left(x_{g-2}\right)$ by the commutativity of the diagram, and this implies $x_{g-2}=0$ by induction. Therefore we can assume that $v \in Y_{g-2}$, hence $v$ is a linear combination of vectors of the form $t_{I} \otimes s_{j}$ where $g-2, g-1, j \notin I$. 
Repeat the procedure, i.e. project from the points $P_{r}, r=g-3 \ldots l$. This can be done since $l=g-3-p \geq k+1$. In this way we can reduce to prove injectivity for the restriction of the map $F_{l}$ to the subspace $Y_{l}$ of $W$ generated by the elements of the form $t_{I} \otimes s_{j}$ where $l, \ldots, g-1, j \notin I$. Observe that since $|I|=l$, we have $Y_{l}=0$, so $F_{l}$ is injective and the theorem is proved.

If $g=2 k$ the proof is analogous: we subsequently project from the points $P_{1}, P_{2}, \ldots, P_{g-l}$. As before note that this can be done since $g-l=p+3 \leq k$. In this way we reduce to prove injectivity for the restriction of the map $F_{l}$ to the subspace $Y$ of $W$ generated by the elements of the form $t_{I} \otimes s_{j}$ where $1,2,3, \ldots, g-l, j \notin I$ and since $|I|=l$, we have $Y=0$, so $F_{l}$ is injective and the theorem is proved.

Corollary 3.3. If the Prym-Green conjecture is true for a Prym-canonical binary curve of genus $g=2 k$ as in (3), then it is true for a Prym-canonical binary curve of genus $g=2 k+1$ as in (3) for generic parameters $a_{i, j}$.

Proof. The conjecture for $g=2 k+1$ says that $K_{k+1,0}\left(C, K_{C}, K_{C} \otimes A\right)=0$, or analogously that property $N_{k-3}$ holds for a generic $C$ embedded with $K_{C} \otimes A$. Hence the corollary immediately follows from Theorem (3.2) with $i=k-3$.

Corollary 3.4. The generic Prym-canonical curve of genus $g$ satisfies property $N_{0}$ for $g \geq 6, N_{1}$ for $g \geq 9, N_{2}$ for $g \geq 10, N_{3}$ for $g \geq 12, N_{4}$ for $g \geq 14$.

Proof. With a direct computation one verifies the Prym-Green conjecture for explicit examples of Prymcanonical binary curves as in (3) for $g=6,9,10,12,14$, so the proof follows from Theorem (3.2) for generic Prym-canonical binary curves, and then by semicontinuity for generic Prym-canonical smooth curves.

To do the computations we wrote a very simple maple code (http://www-dimat.unipv.it/ frediani/prym-can) in which we explicitly give the matrix representing the Koszul map $F_{l}$ : for every multi-index $J$ with $|J|=l-1$, we take the projection of the image of $F_{l}$ onto $t_{J} \otimes B_{g}$ and we restrict it to the rational components $C_{j}$. So we have two polynomials in one variable and we take their coefficients.

Once the matrix is constructed, for $g=6,9,10,12$, maple computed its rank modulo 131, which turned out to be maximal. In the case $g=14$ the order of the matrices was too big, so Riccardo Murri made the rank computation using the Linbox ([14]) and Rheinfall ([16]) free software libraries. Two different rank computation algorithms were used: Linbox" "black box" implementation of the block Wiedemann method ([13, 19]), and Rheinfall's Gaussian Elimination code([15]). Results obtained by either method agree.

In both cases, the GNU GMP library ([10]) provided the underlying arbitrary-precision representation of rational numbers and exact arithmetic operations.

Remark 3.5. For Prym-canonical curves of genus 8, the maple computation on specific examples of binary curves gives $\operatorname{dim} K_{1,2}\left(C, K_{C} \otimes A\right)=1$. This result is compatible with the computations in [5].

For Prym-canonical binary curves of genus 16, we constructed the matrix representing the Koszul map $F_{8}$ on examples using maple and Riccardo Murri computed its rank as explained in the proof of Corollary (3.4). Again it turned out that $\operatorname{dim} K_{5,2}\left(C, K_{C} \otimes A\right)=1$, confirming the computations in [5].

\section{Property $N_{p}$ For canonical binary Curves}

In analogy with the Prym-canonical case, we study now property $N_{p}$ for canonical binary curves with the same inductive method, projecting from a node. So, let $C \subset \mathbb{P}^{g-1}$ be a canonical binary curve and denote by $\tilde{C}_{r}$ the partial normalization of $C$ at the node $P_{r}, 1 \leq r \leq g$. As above, for a general choice of the $a_{i, j}$ 's, the projection from $P_{r}$ sends the curve $C$ to the canonical model of $\tilde{C}_{r}$ in $\mathbb{P}^{g-2}$, where $\tilde{C}_{r}$ is parametrized by $a_{i, j}^{\prime}=a_{i, j}$ for $i \leq r-1, j=1,2, a_{i, j}^{\prime}=a_{i+1, j}$ for $i \geq r, j=1,2$.

Set $H_{g}:=H^{0}\left(C, \omega_{C}\right), D_{g}:=H^{0}\left(C, \omega_{C}^{2}\right), F_{l}: \Lambda^{l} H_{g} \otimes H_{g} \rightarrow \Lambda^{l-1} H_{g} \otimes D_{g}$ the Koszul map. Denote as before by $\left\{s_{1}, \ldots, s_{g}\right\}$ the basis of $H_{g}$ given by the coordinate hyperplane sections in $\mathbb{P}^{g-1} \cong \mathbb{P}\left(H_{g}^{\vee}\right)$. 
$H_{g-1, r}:=H^{0}\left(\tilde{C}_{r}, \omega_{\tilde{C}_{r}}\right), D_{g-1, r}:=H^{0}\left(\tilde{C}_{r}, \omega_{\tilde{C}_{r}}^{2}\right)$. Denote by $\left\{s_{1}^{\prime}, \ldots, s_{g-1}^{\prime}\right\}$ the basis of $H_{g-1, r}$ given by the coordinate hyperplane sections in $\mathbb{P}^{g-2} \cong \mathbb{P}\left(H_{g-1, r}^{\vee}\right)$.

We have the injections $H_{g-1, r} \stackrel{J_{r}}{\longrightarrow} H_{g}$, as in (6) and $D_{g-1, r} \stackrel{L_{r}}{\longrightarrow} D_{g}$, which on the set of generators of $B_{g-1, r}$ given by $s_{i}^{\prime} s_{j}^{\prime}, i, j=1 \ldots g-1$, is given by $s_{i}^{\prime} s_{j}^{\prime} \mapsto J_{r}\left(s_{i}^{\prime}\right) J_{r}\left(s_{j}^{\prime}\right)$.

We finally have a map

$$
\Lambda^{l-1} H_{g-1, r} \stackrel{\wedge s_{r}}{\longrightarrow} \Lambda^{l} H_{g},
$$

where by $\wedge s_{r}$ we indicate the composition of the natural map induced by $J$ at the level of the $l$ - 1-th exterior power $\Lambda^{l-1} H_{g-1, r} \rightarrow \Lambda^{l-1} H_{g}$ composed by the wedge product with $s_{r}, \Lambda^{l-1} H_{g} \stackrel{\wedge s_{r}}{\longrightarrow} \Lambda^{l} H_{g}$.

We are interested in property $N_{p}$ for these curves, hence by duality, in the vanishing of $K_{g-2-p, 1}\left(C, K_{C}\right)$. Clearly the vanishing of $K_{l, 1}\left(C, K_{C}\right)$ is equivalent to the injectivity of the map

$$
\frac{\Lambda^{l} H_{g} \otimes H_{g}}{\Lambda^{l+1} H_{g}} \rightarrow \Lambda^{l-1} H_{g} \otimes D_{g}
$$

coming from the Koszul complex.

Notice that there is an isomorphism between $\frac{\Lambda^{l} H_{g} \otimes H_{g}}{\Lambda^{l+1} H_{g}}$ and the subspace $V_{g}$ of $\Lambda^{l} H_{g} \otimes H_{g}$ generated by the elements of the form $s_{I} \otimes s_{j}$, where $j \geq i_{1}$, so the above injectivity is equivalent to the injectivity of the restriction of $F_{l}$ to $V_{g}$.

We have the following commutative diagram

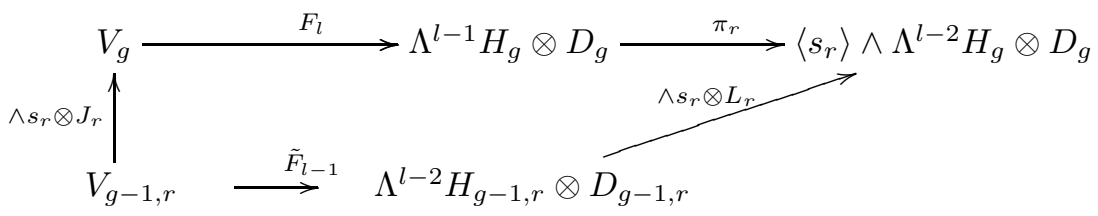

where $V_{g-1, r}$ is the subspace of $\Lambda^{l-1} H_{g-1, r} \otimes H_{g-1, r}$ generated by the elements of the form $s_{J}^{\prime} \otimes s_{j}^{\prime}$, where $j \geq j_{1}$.

Let $W \subset V_{g, l}$ be the subspace generated by the elements of the form $s_{I} \otimes s_{j}$, where $j \notin I$ and $j \geq i_{1}$.

Remark 4.1. The map $F_{l}: V_{g} \rightarrow \Lambda^{l-1} \otimes B_{g}$ is injective if and only if $F_{l \mid W}$ is injective.

Proof. The proof is completely analogous to the proof of (3.1).

Theorem 4.2. If property $N_{p}$ holds for a canonical binary curve of genus $g-1$ as in (4.2), then the same property holds for a canonical binary curve of genus $g$ as in (4.2) for a generic choice of the parameters.

Proof. From the above discussion we know that the statement is equivalent to prove injectivity of the Koszul map $F_{l}: V_{g} \rightarrow \Lambda^{l-1} H_{g} \otimes D_{g}$ for $l=g-2-p$, while by assumption we know injectivity of the map $\tilde{F}_{l-1}: V_{g-1, r} \rightarrow \Lambda^{l-2} H_{g-1, r} \otimes D_{g-1, r}$.

We first project from $P_{g}$. By Remark (4.1) we can reduce to prove injectivity of $F_{l}$ restricted the subspace $W$ generated by such $s_{I} \otimes s_{j}$ with $j \notin I, j>i_{1}$. Note that as before we can decompose $W$ as $W:=X_{g} \oplus Y_{g}$, where $X_{g}$ is the intersection with $W$ of the image of the map $\wedge s_{g} \otimes J_{g}$ in diagram (13) and $Y_{g}$ is the subspace of $W$ generated by such $s_{I} \otimes s_{j}$ with $g \notin I$ and $j \notin I, j>i_{1}$ :

$$
X_{g}=\left\langle s_{g} \wedge s_{J} \otimes s_{j} \mid j \notin J, j>j_{1}\right\rangle, Y_{g}=\left\langle s_{I} \otimes s_{j} \mid j, g \notin I, j>i_{1}\right\rangle
$$

If $F_{l}\left(x_{g}+y_{g}\right)=0$, where $x_{g} \in X_{g}, y_{g} \in Y_{g}$, then $0=\pi_{g} \circ F_{l}\left(x_{g}+y_{g}\right)=\pi_{g} \circ F_{l}\left(x_{g}\right)=\left(\wedge s_{g} \otimes L_{g}\right) \circ$ $\tilde{F}_{l-1}\left(x_{g}\right)$. Hence $x_{g}=0$, since by induction $\tilde{F}_{l-1}$ is injective. So we have reduced to prove injectivity of $F_{l}$ restricted to $Y_{g}$. 
Repeat the procedure, i.e. project from the points $P_{r}, r=g-1 \ldots l$. In this way we can reduce to prove injectivity for the restriction of the map $F_{l}$ to the subspace $Y_{l}$ of $W$ generated by the elements of the form $s_{I} \otimes s_{j}$ where $l, \ldots, g, j \notin I, j>i_{1}$. Observe that since $|I|=l$, we have $Y_{l}=0$, so $F_{l}$ is injective and the theorem is proved.

Remark 4.3. Notice that, by the theorem of Green and Lazarsfeld (111]), if $p>g-\left[\frac{g}{2}\right]-2$, condition $N_{p}$ does not hold for any curve $\tilde{C}$ of genus $g-1$.

Corollary 4.4. If the Green conjecture is true for a canonical binary curve of genus $g=2 k-1$ as in (4.2), then it is true for a canonical binary curve of genus $g=2 k$ as in (4.2) for a generic choice of the parameters.

Proof. The conjecture for $g=2 k$ says that $K_{k, 1}\left(C, K_{C}\right)=0$, or analogously that property $N_{k-2}$ holds for $C$ embedded with $K_{C}$. By assumption we know that $K_{k-1,1}\left(\tilde{C}, K_{\tilde{C}}\right)=0$, namely that property $N_{k-2}$ holds for $\tilde{C}$ embedded with $K_{\tilde{C}}$, so the thesis immediately follows from (4.2) .

With maple (http://www-dimat.unipv.it/ frediani/greenfinal.tar.gz) one verifies the conjecture for $g=5,7,9,11$, so one can prove with the same method that if $g \geq 3$, then propery $N_{0}$ holds (see also [3] section 2), if $g \geq 5$, then propery $N_{1}$ holds, if $g \geq 7$, then propery $N_{2}$ holds, and if $g \geq 9$, then property $N_{3}$ holds, and if $g \geq 11$, then property $N_{4}$ holds.

\section{REFERENCES}

[1] M. Aprodu, G. Farkas. Green's Conjecture for curves on arbitrary K3 surfaces. Compositio Math. 147 (2011) 839-851.

[2] M. Aprodu and J. Nagel, Koszul cohomology and algebraic geometry, University Lecture Series, Vol. 52, American Mathematical Society 2010.

[3] Calabri, A., Ciliberto, C., Miranda, R., The rank of the 2nd Gaussian map for general curves, Michigan Math. J. 60 (2011), no. 3, 545559 .

[4] Caporaso, L., Brill-Noether theory of binary curves. Mathematical Research Letters - Volume 17 - Issue 2/ March 2010 pp. $243-262$.

[5] Alessandro Chiodo, David Eisenbud, Gavril Farkas, Frank-Olaf Schreyer, Syzygies of torsion bundles and the geometry of the level 1 modular variety over $M_{g}$. arXiv:1205.0661 1 . To appear in Inventiones Math.

[6] Colombo, E., Frediani, Prym map and second gaussian map for Prym-canonical line bundles. arXiv:1105.4472 v1. Advances in Mathematics (2013), http://dx.doi.org/10.1016/j.aim.2013.02.009

[7] O. Debarre, Sur le Probleme de Torelli pour les Varietes de Prym. American Journal of Mathematics, Vol. 111, No. 1 (1989), pp. 111-134.

[8] Farkas, Gavril; Ludwig, Katharina, The Kodaira dimension of the moduli space of Prym varieties. J. Eur. Math. Soc. (JEMS) 12 (2010), no. 3, 755-795.

[9] Franciosi, Marco, Adjoint divisors on algebraic curves. Appendix A by Fabrizio Catanese. Adv. Math. 186 (2004), no. 2,317333 .

[10] GNU GMP web site: http://www.gmplib.org/

[11] M. Green, Koszul cohomology and the cohomology of projective varieties, Journal of Differential Geometry 19 (1984), 125-171.

[12] A. Hirschowitz, S. Ramanan. New evidence for Green's conjecture on syzygies of canonical curves. Ann. Sci. École Norm. Sup. 31 (1998) 145-152.

[13] E. Kaltofen and B. D. Saunders. On Wiedemanns method of solving sparse linear systems. In: Applied Algebra, Algebraic Algorithms and Error-Correcting Codes, volume 539 of LNCS, pages 2938, Springer, 1991.

[14] Linbox web site: http://linalg.org/

[15] R. Murri, A novel parallel algorithm for Gaussian Elimination of sparse unsymmetric matrices, in: Parallel Processing and Applied Mathematics 9th International Conference (PPAM 2011), Torun, Poland, September 11-14, 2011. Revised Selected Papers, Part I, volume 7203 of LNCS, Springer 2012.

[16] Rheinfall web site: http://rheinfall.googlecode.com/

[17] F.-O. Schreyer, Green's conjecture for general $p$-gonal curves of large genus. Algebraic curves and projective geometry (Trento, 1988), 254-260, Lecture Notes in Math., 1389, Springer, Berlin, 1989.

[18] M. Teixidor i Bigas, Green's conjecture for the generic $r$-gonal curve of genus $g \geq 3 r-7$. Duke Math. J. 111 (2002) 195-222.

[19] W. J. Turner, Black box linear algebra with the LINBOX library, Ph.D. Thesis, University of North Carolina, 2002.

[20] C. Voisin, Green's generic syzygy conjecture for curves of even genus lying on a K3 surface. J.European Math. Soc. 4 (2002), 363-404.

[21] C. Voisin, Green's canonical syzygy conjecture for generic curves of odd genus, Compositio Math. 141 (2005), 11631190 . 
Dipartimento di Matematica, Università di Milano, via Saldini 50, I-20133, Milano, Italy

E-mail address: elisabetta.colombo@unimi.it

Dipartimento di Matematica, Università di Pavia, via Ferrata 1, I-27100 Pavia, Italy

E-mail address: paola.frediani@unipv.it 